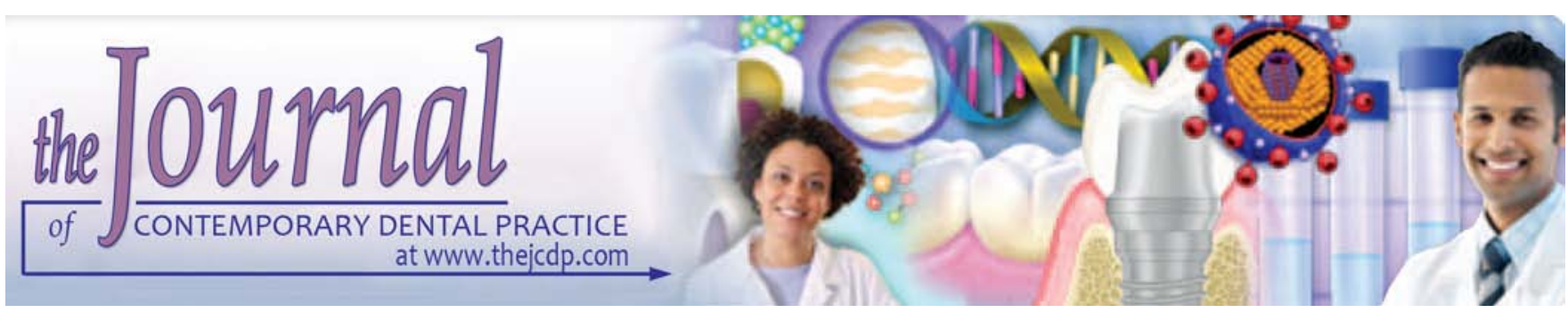

\title{
Influence of Head Orientation in Linear Measurement for Implant Planning in Cone Beam Computed Tomography
}

\author{
${ }^{1}$ Abbas Shokri, ${ }^{2}$ Samira Khajeh, ${ }^{3}$ Atefeh Khavid, ${ }^{4}$ Sara Tabari, ${ }^{5}$ Shiva Yarmohammadi
}

\begin{abstract}
Aim: Use of dental implants in edentulous patients has become a common treatment modality. Treatment of such implants requires radiographic evaluation, and in most cases several different imaging techniques are necessary in order to evaluate the height, width and the structure of bone at implant site. The aim of this study was to evaluate the influence of head orientation in linear measurement for implant planning in cone beam computed tomography (CBCT).
\end{abstract}

Materials and methods: In the present in vitro study, 11 human dry mandibles were used. The width and height of bone at the central, canine and molar teeth areas were measured on the left and right sides by using digital calipers (as gold standard) and on CBCT images with Reaxis option and no Reaxis. Data were analyzed with Statistical package for the social sciences (SPSS) 18, using pair t-test, Tukey test and intraclass correlation (ICC).

Results: Data were collected by evaluation of 11 skulls and 66 samples on the whole. There were no significant differences in bone width in any area in both Reaxis and no Reaxis option $(p>0.05)$. There were significant differences in bone height in the central $(p=0.005)$ and molar teeth areas $(p=0.010)$. The results did not show any significant differences between the observers $(p=0.329)$.

Conclusion: Using the Reaxis option does not affect the measurement of bone width, while use of this option can make a significant difference for height measurements.

Keywords: Bone width, Bone height, СВСТ, Head orientation, Reaxis option, Reslice option.

How to cite this article: Shokri A, Khajeh S, Khavid A, Tabari S, Yarmohammadi S. Influence of Head Orientation in Linear

\footnotetext{
1-5Department of Oral and Maxillofacial Radiology, Dental School of Hamadan University of Medical Sciences, Hamadan Iran
}

Corresponding Author: Atefeh Khavid, Postgraduate Student Department of Oral and Maxillofacial Radiology, Dental School of Hamadan University of Medical Sciences, Hamadan, Iran Phone: +98-9188362065, e-mail: atefehkhavid@gmail.com
Measurement for Implant Planning in Cone Beam Computed Tomography. J Contemp Dent Pract 2015;16(7):542-546.

Source of support: Nil

Conflict of interest: None

\section{INTRODUCTION}

Use of dental implants has increased in recent years in the treatment of edentulous patients. Such treatment modalities require extensive radiographic evaluations, and in most cases different imaging techniques are necessary in order to evaluate the height, width and structure of bone at implant placement site. ${ }^{1,2}$

Analyses carried out onconventional radiographic images are, in fact, carried out on a two-dimensional (2D) image of a three-dimensional (3D) structure. It is difficult to carry out measurements on images and it is associated with some errors, which can be attributed to image magnification and distortion. ${ }^{3}$ Measurement accuracy of imaging techniques has a great role in the success of implant treatment, therefore, systems with the highest accuracy are preferable for imaging techniques for implant placement.

Cone beam computed tomography (CBCT) is a newer technique that is both inexpensive and small enough to be used in the dental office, which yields high-resolution images with favorable accuracy. Therefore, it is increasingly used to evaluate different jaw areas and measurements. $^{4,5}$

Cone beam computed tomography can provide sub millimeter spatial resolution for images of the craniofacial complex, with scanning time comparable to panoramic radiography. The cone beam technique uses rotational scanning of an X-ray source, reciprocating an X-ray detector around the patient head. The CBCT software provides tools to measure distances, angles, zoom, invert the gray scale, adjust contrast and gamma changes. 6,7 
Head and jaw position is important in radiographic imaging, numerous articles have highlighted the problems caused by the incorrect positioning of patients while obtaining panoramic radiographs, especially those that are to be used for implant planning. ${ }^{8}$ Changes in aspects, such as the configuration of the dental arch and distortions in the inclination of a tooth can arise, and errors in the linear measurements for preoperative planning can occur. ${ }^{8-10}$

Though the CBCT is an examination method that is most frequently indicated for the planning of implants, few studies have investigated the real effects of the incorrect positioning of the patient during image acquisition or the influence of positioning variations on the measurement of the height and width of the remaining alveolar bone. ${ }^{11-14}$

In imaging with $\mathrm{CBCT}$ systems, mandibular plane must be paralleled with horizon, and in some patients, such as patient with short neck or respiratory complication, creation a correct position of head is difficult. In these cases, the mandibular plane should be modified after imaging by using software options, such as reorientation and Reslice mode. Therefore, the objective of the present study was to evaluate the influence of head orientation in linear measurement for implant planning in CBCT by using ReAxis (Reslice) option and without this.

\section{MATERIALS AND METHODS}

Eleven dry human mandibles were used in the present study. The mandibles had no fractures, severe deformities and severe resorption. The following areas underwent measurements:

- Two anterior areas of the left and right central teeth

- Two middle areas of the left and right canine teeth

- Two posterior areas of the left and right first molar teeth.

In these areas, two measurements were made as follow:

- The height of the mandible

- The buccolingual thickness of the mandible.

A digital caliper (Mituyoto, Japan) was used as the gold standard for physical measurements for greater reliability of the study, in a manner similar to that in other studies. The maximum buccolingual diameter which could be measured with the caliper without interference with bony undercuts was designated as the diameter reference, and the maximum distance between the buccal margin of the socket and the external border of the inferior cortex was designated as a reference for height; these references were marked. To prevent placement of the selected areas in an undercut, firstly the points were selected in a manner so that the tips of the two arms of the digital caliper were exactly placed opposite to each other, and then these points were marked. Measurements with the caliper were carried out at this stage; two observers measured all the predetermined areas in all the samples and registered the data in special forms. Then, the means of the measurements made by the two observers were calculated as the gold standard. Barium sulfate radiopaque markers were placed on the points by a very small burnisher, so that the points would be visible on radiographic images. In order to reduce radiopaque marker artifact, a spacer was placed on the marked areas by the markers, using rose wax, with a thickness of $1 \mathrm{~mm}$. Sticky wax was placed on the markers so that they would not be detached.

\section{Imaging of the Mandibles}

Imaging techniques were carried out by Cranex 3D X-ray machine (Soredex, Finland) at $90 \mathrm{kVp}, 5 \mathrm{~mA}$ and 12.6 seconds. A sponge with $20^{\circ}$ gradient was made and mandibles were placed on this sponge for similar gradient, and imaging were taken (Fig. 1).

Then the images were reconstructed by the special software program (Cranex 3D X-ray machine), with the proprietary name of 'ON Demand 3D Dental', at $0.5 \mathrm{~mm}$ slice thicknesses. In order to evaluate the effect of head orientation on the accuracy of linear measurements, images were reconstructed in two formats: Slice and Reslice option. With Reslice option, we can change the primary head position for having the correct position (Fig. 2).

The special ruler of the software program was selected and the areas were measured in all the 11 mandibles and data were registered in special checklists.

All the images were evaluated by two observers, an oral and maxillofacial radiologist and a postgraduate student of oral and maxillofacial radiography, twice in an interval of 2 weeks.

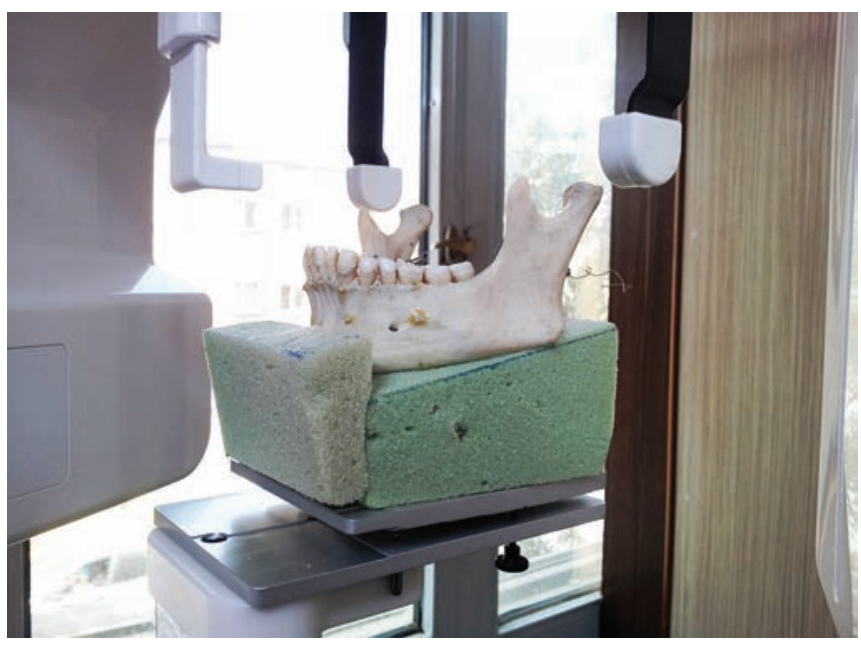

Fig. 1: For similar gradient, mandibles were placed in sponge with $20^{\circ}$ gradient 

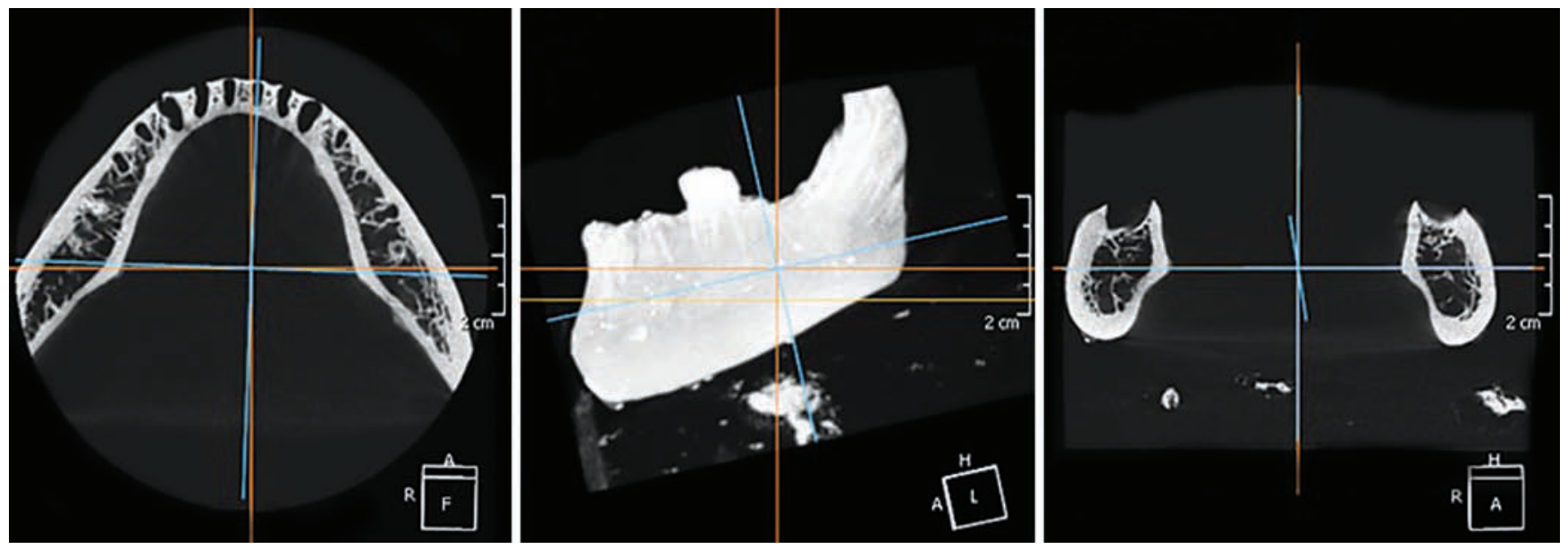

Fig. 2: Using the reslice option for changing the jaw orientation

\section{Data Acquisition}

After collection of the necessary data, they were entered into SPSS 16 software program. Intraclass correlation (ICC) was used to evaluate interexaminer agreement. This index was calculated separately for dimensions and for different options of the X-ray machine. The t-test was used to compare the means of the measurements made with the gold standard. In order to analyze measurement errors, the absolute values of the differences between each measurement and the gold standard were calculated. Repeated measures analysis of variance (ANOVA) was used to evaluate the cumulative effect of the machine's options, measurements, measurement locations and measurement accuracy, and the interactive effect of the variables. Tukey's Honestly significant difference (HSD) test was used for two-by-two comparisons. All the statistical tests were carried out at a significance level of 0.05 .

\section{RESULTS}

The data in the present study were collected by evaluating measurements made in relation to the thickness and height of bone in 11 mandibles in different tooth areas of central, canine and molar teeth on the right and left sides. On the whole, there were 66 measurements which were made by two observers using CBCT machine in Reslice option and without this.

Table 1 presents the results of comparisons made between the mean bone widths and heights in all the areas as measured by the two observers using a digital caliper and CBCT images, using Reslice option and without this.

Based on Table 1, the differences were not significant between the mean values measured on CBCT images, while the gold standard values were significant $(\mathrm{p}<0.05)$. Table 2 presents multiple comparisons at central, canine and molar areas in relation to the width of bone using CBCT machine at $0.5 \mathrm{~mm}$ with and without Reslice options. Based on Table 2, the differences between the measurements in relation to the width of bone were not significant $(p>0.05)$. Table 3 presents multiple comparisons at central, canine and molar areas in relation to the height of bone using CBCT machine at $0.5 \mathrm{~mm}$ with and without Reslice options. Based on Table 3, the differences between the measurements in relation to the height of bone were significant in incisor and molar areas $(p<0.05)$, but not in canine area $(\mathrm{p}>0.05)$.

\section{Agreement between Observers}

Regarding the statistical analysis, no statistically significant difference was seen in the interobserver reliability $(p=0.329)$. The ICC for interobserver reliability varied from 0.996 to 0.999 . The statistical analysis showed high interobserver reliability $(\mathrm{p}<0.05)$.

\section{DISCUSSION}

Provision of dental implants for patients who have lost their teeth is a common practice. Anatomic structures and the surrounding bone must be assessed both clinically

Table 1: Comparisons between the mean bone widths and heights in all the areas using a digital caliper and CBCT images using reslice option and without this

\begin{tabular}{|c|c|c|c|c|c|c|}
\hline & \multicolumn{2}{|c|}{$\begin{array}{l}\text { Mean difference in molar area } \\
(\mathrm{mm})\end{array}$} & \multicolumn{2}{|c|}{$\begin{array}{l}\text { Mean difference in canine area } \\
(\mathrm{mm})\end{array}$} & \multicolumn{2}{|c|}{$\begin{array}{l}\text { Mean difference in incisor are } \\
(\mathrm{mm})\end{array}$} \\
\hline & Height & Width & Height & Width & Height & Width \\
\hline Without reslice option & 0.858 & 0.278 & 0.468 & 0.251 & 0.388 & 0.289 \\
\hline With reslice option & 0.437 & 0.320 & 0.560 & 0.216 & 0.512 & 0.264 \\
\hline
\end{tabular}


Table 2: Comparisons between the mean bone widths in all the areas using reslice option and without this

\begin{tabular}{llll}
\hline Area & Mean difference & Standard deviation & $p$-value \\
\hline Incisor & 0.056 & 0.279 & 0.351 \\
Canine & 0.048 & 0.274 & 0.415 \\
Molar & 0.008 & 0.219 & 0.891 \\
\hline Total & 0.041 & 0.260 & 0.225 \\
\hline
\end{tabular}

Table 3: Comparisons between the mean bone height in all the areas using reslice option and without this

\begin{tabular}{lcll}
\hline Area & Mean difference & Standard deviation & $p$-value \\
\hline Incisor & 0.312 & 0.471 & 0.005 \\
Canine & 0.028 & 0.624 & 0.831 \\
Molar & -0.605 & 0.918 & 0.010 \\
\hline Total & -0.063 & 0.771 & 0.517 \\
\hline
\end{tabular}

and radiographically before placing implants. ${ }^{1}$ Imaging options began with 2D imaging and now include 3D imaging techniques. Diagnostic information, treatment planning and outcome benefits have increased with the use of 3D imaging techniques. Using 3D virtual planning techniques before treatment has resulted in optimal implant placement and improved clinical results. ${ }^{15,16}$

Conventional radiography, such as panoramic and periapical radiographs do not provide cross-sectional information and are, therefore, insufficient for implant site evaluation. Tomographic images are useful for assessing information on ridge measurements three-dimensionally, considered essential for the surgical planning of implant placement. ${ }^{17-19}$

Cone beam computed tomography provides a valuable tool for evaluating craniofacial region. Effective radiation dose from a scan of maxillofacial volume is significantly lower than medical CT and is in the range of conventional dental radiographies. ${ }^{20}$

It is widely reported in the literature that variations in the positioning of the patient during a radiographic examination produce dimensional variations in the images.

Poor positioning of the patient can exacerbate the distortions and enlargements of the structures, thus interfering with the planning of the placement of dental implants. ${ }^{9}$

Furthermore, the use of image-based examination is increasing with the increased performance of so-called 'virtually guided surgeries'. In these cases, the planning is completely dependent on the quality of the image acquired. $^{21}$

The incorrect acquisition of the image can result in severe mistakes in the execution of the proposed treatment. ${ }^{12}$ Few studies have addressed the influence of the positioning of the patient on tomographic examinations for the projection of implants. This fact is emphasized when dealing with examinations based on CBCT.
In imaging with CBCT systems, mandibular plane must be paralleled with horizon, and in some patients, such as patient with short neck or respiratory complication, creation of a correct position of head is difficult. In these cases, the mandibular plane should be modified after imaging by using software options, such as reorientation and Reslice mode. Therefore, the objective of the present study was to evaluate the influence of head orientation in linear measurement for implant planning in CBCT by using ReAxis (Reslice) option and without this.

What differentiates the present study from previous studies is the use of a spacer and barium sulfate marker in measurements, which prevented streak artifact in the external surface of the cortical bone. Metallic radiopaque markers were not used in the present study because they produce metal artifacts, and decrease image quality at areas undergoing measurement. In order to prevent superimposition of the opacity of the marker on the cortical bone so that the area involved can be measured without the thickness of the marker, in previous studies small metallic balls or orthodontic wines have been directly placed on bone. However, they undermine the accuracy of measurements made in these studies because they produce severe metal artifacts. In addition, use of gutta-percha is controversial due to its dimensional instability and low opacity. ${ }^{22}$ Another advantage of the present study was the use of a sponge with $20^{\circ}$ gradient on which the mandibles were placed for similar gradient in all imaging.

Results showed that there were no significant differences in bone width in any area in both Reaxis (Reslice) and no Reaxis (Reslice) options ( $p>0.05)$. There were significant differences in bone height in the central $(\mathrm{p}=$ $0.005)$ and molar teeth areas $(\mathrm{p}=0.010)$.

These results can be explained by the fact that the crest of the alveolar ridge, especially in the anterior area, is not covered with compact bone and it is difficult to determine its exact location by a radiographic technique. In addition, the shapes and gradients of the buccal and lingual tables (the shape of the mandibular arch) are different in the anterior and posterior areas. Therefore, it is more probable for markers not to be opposed in a crosssection in the anterior area as compared to the posterior area. This issue increases the odds of measurement errors, although the OnDemand 3D software can make 3D measurements, i.e. it is possible to determine one point in one cross-section and determine the second point in other consecutive cross-sections, so that the software program can measure the linear distance between the two points. However, Sheikhi et al did not report any significant differences between the central, premolars 
and molar areas, contrasting to the results of the present study. ${ }^{23}$ Such a difference might be attributed to the type of CBCT machine used, and therefore, the capabilities of their software programs were different in the two studies.

Dantas et al evaluated the influence of superiorinferior positioning on the measurements of bone height and width from multi-slice CT images. The authors noticed that for some cuts in the orthoradial image, principally those in the premolar region, at the site of what appeared to be the anatomical repair corresponding to the area studied, the radiopaque marker was not present.

In conclusion, we can understand from this study that using the Reaxis (Reslice) option does not affect the measurement of bone width, while use of this option can make a significant difference in height measurements.

\section{CONCLUSION}

We can conclude that alterations in the positioning of the patient during the acquisition of CBCT images can lead to variations in the measurements of bone height and width, possibly compromising the treatment and even causing damage to important anatomical structures. Therefore, it is essential that standardization of the positioning at the moment of acquisition as well as the use of an appropriate protocol, be performed routinely in the radiology clinic.

\section{ACKNOWLEDGMENT}

The authors would like to extend their gratitude to the deputy of research at Hamadan University of medical sciences and the dental research center for the support provided.

\section{REFERENCES}

1. Miller CS, Nummikoski PV, Barnett DA, Langlais RA. Crosssectional tomography. Oral Surg Oral Med Oral Pathol 1990;70(6):791-797.

2. White SC, Pharoah MJ. Oral radiology: principles and interpretations. 5th ed. Cone-beamed computed tomography: anatomy St Louis: Elsevier; 2004. Chapter 13, p. 214-228.

3. Stavropoulos A, Wenzel A. Accuracy of cone beam dental CT, intraoral digital and conventional film radiography for detection of periapical lesions. An ex vivo study in pig jaws. Clin Oral Invest 2007;11(1):101-106.

4. Nickenig H, Wichmann M, Hamel J, Schlegel KA, Eitner S. Evaluation of the difference in accuracy between implant placement by virtual planing data and surgical guied templates versus the conventional free-hand method-a combined in vivo-in vitro techniques using cone beam CT (part II). J Craniomaxillofac Surg 2010;38(7):488-493.

5. Alqerban A, Jacobs R, Couto Souza P, Willems G. In vitro comparison of 2 cone beam systems and panoramic imaging for detecting simulated canine impaction-induced external root resorption in maxillary lateral incisor. Am J Orthod Dentofac Orthop 2009;136(6):764.e1-e11.
6. Fatemitabar SA, Nikgoo A. Multichannel computed tomography versus cone-beam computed tomography: linear accuracy of in vitro measurements of the maxilla for implant placement. Int J Oral Maxillofac Implants 2010;25(3):499-505.

7. Pinsky HM, Dyda S, Pinsky RW, Misch KA, Sarment DP. Accuracy of three-dimensional measurements using conebeam CT. Dentomaxillofac Radiol 2006;35(6):410-416.

8. Liang H, Frederiksen NL. Focal trough and patient positioning. Dentomaxillofac Radiol 2004;33(2):128-129.

9. Hardy TC, Suri L, Stark P. Influence of patient head positioning on measured axial tooth inclination in panoramic radiography. J Orthod 2009;36(2):103-110.

10. Dantas JA, Montebello Filho A, Campos PS. Computed tomography for dental implants: the influence of the gantry angle and mandibular positioning on the bone height and width. Dentomaxillofac Radiol 2005;34(1):9-15.

11. Ludlow JB, Laster WS, See M, Bailey LJ, Hershey HG. Accuracy of measurements of mandibular anatomy in cone beam computed tomography images. Oral Surg Oral Med Oral Pathol Oral Radiol Endod 2007;103(4):534-542.

12. Visconti M, Verner FS, Assis NM, Devito KL. Influence of maxillomandibular positioning in cone beam computed tomography for implant planning. Int J Oral Maxillofac Surg 2013;42(7):880-886.

13. Hassan B, van der Stelt P, Sanderink G. Accuracy of three dimensional measurements obtained from computed tomography surface-rendered images for cephalometric analysis: influence of patient scanning position. Eur J Orthod 2009;31(2):129-134.

14. Lund H, Grondahl K, Grondahl HG. Accuracy and precision of linear measurements in cone beam computed tomography Accuitomo tomograms obtained with different reconstruction techniques. Dentomaxillofac Radiol 2009;38(6):379-386.

15. Hatcher DC, Dial C, Mayorga C. Cone beam CT for presurgical assessment of implant sites. J Calif Dent Assoc 2003;31(11):825-833.

16. Quirynen M, Lamoral Y, Dekeyser C, Peene P, van Steenberghe D, Bonte J, Baert AL. CT scan standard reconstruction technique for reliable jaw bone volume determination. Int J Oral Maxillofac Implants 1990;5(4):384-389.

17. Lecomber AR, Downes SL, Mokhtari M, Faulkner K. Optimisation of patient doses in programmable dental panoramic radiography. Dentomaxillofac Radiol 2000; 29(2):107-112.

18. Danforth RA, Dus I, Mah J. 3D volume imaging for dentistry: A new dimension. J Calif Dent Assoc 2003;31(11):817-823.

19. Jeffcoat M, Jeffcoat RL, Reddy MS, Berland L. Planning interactive implant treatment with 3-D computed tomography. J Am Dent Assoc 1991;122(11):40-44.

20. Brown AA, Scarfe WC, Scheetz JP, Silveira AM, Farman AG. Linear accuracy of cone-beam CT derived 3D images. Angle Orthod 2009;79(1):150-157.

21. Ghanai S, Marmulla R, Wiechnik J, Muhling J, Kotrikova B. Computer-assisted three-dimensional surgical planning: 3D virtual articulator: technical note. Int J Oral Maxillofac Surg 2010;39(1):75-82.

22. Loubele M, Bogaerts R, Dijck EV, Pauwels R, Vanheusden $S$, Suetens P, Marchal G, Sanderink G, Jacobs R. Comparison between effective radiation dose of CBCT and MSCT scanners for dentomaxillofacial applications. Eur J Radiol 2009;71 (3):461-468.

23. Sheikhi M, Ghorbanizadeh S, Abdinian M, Goroohi $\mathrm{H}$, Badrian H. Accuracy of Linear Measurements of Galileos Cone Beam Computed Tomography in Normal and Different Head Positions. Int J Dent 2012;2012:Article ID 214954. 